\title{
Clinical Evaluation of Membrane Excitability in Muscle Channel Disorders: Potential Applications in Clinical Trials
}

\author{
James C. Cleland and Eric L. Logigian \\ University of Rochester School of Medicine and Dentistry, Rochester, New York
}

\begin{abstract}
Summary: Muscle channelopathies are inherited disorders that cause paralysis and myotonia. Molecular technology has contributed immeasurably to diagnostic testing, to correlation of genotype with phenotype, and to insight into the pathophysiology of these disorders. In most cases, the diagnosis of muscle channelopathy is still made on clinical grounds, but is supported by ancillary laboratory and electrodiagnostic testing such as serum potassium measurement, exercise testing, repetitive nerve stimulation, needle electromyography, calculation of muscle fiber conduction velocity, or electromyography power spectra. Although provocative glucose or potassium challenges are now infrequently performed, they have contrib-
\end{abstract}

uted greatly to our understanding of the pathophysiology of these disorders, and to our ability to differentiate between periodic paralysis types. Despite considerable progress, ample opportunity remains for future clinical research, particularly in expanding genotype-phenotype correlations and in optimizing electrodiagnostic methods. With respect to diagnostic testing, there is a need for accurate, efficient, and cost-effective bedside testing, given the substantial proportion (as high as 20\%) of genetically undefined cases. Even in genetically defined cases, minimal clinical expressivity due to incomplete penetrance poses a significant challenge to currently available nonmolecular testing. Key Words: Diagnosis, electromyography, channelopathy.

\section{INTRODUCTION}

Ion channels play a critical role in setting and maintaining the resting membrane potential and controlling excitability of skeletal muscles. Dysfunction of these channels causes the periodic paralysis disorders (PP) (hypokalemic and hyperkalemic types and AndersenTawil syndrome) and the nondystrophic myotonias (recessive and dominant myotonia congenita, paramyotonia congenita, and potassium-aggravated myotonia). In PP, abnormal and persistent membrane depolarization renders the muscle fiber hypoexcitable or inexcitable through inactivation of sodium channels. Early on in the disease, muscle strength returns to normal once the resting membrane potential is restored to normal, but after several years slowly progressive interictal weakness develops, the cause of which remains a mystery. Conversely, in the nondystrophic myotonias abnormal membrane hyperexcitability occurs as a result of more mild membrane depolarizations sufficient to recurrently activate sodium channels. This membrane depolarization is

Address correspondence and reprint requests to: James C. Cleland, MBChB, Consultant Neurologist, Waikato Hospital, Hamilton, New Zealand. E-mail: clelandj@waikatodhb.govt.nz. due to either 1) reduced chloride conductance (myotonia congenita), or 2) altered sodium channel gating kinetics that lead to either persistent inward sodium current, enhanced recovery from inactivation, or both (paramyotonia congenita and potassium aggravated myotonia). Although most individuals with mutations in the skeletal muscle sodium channel exhibit either a predominant myotonic or PP phenotype, some have both, an indication that myotonia and paralysis may be on a physiologic continuum.

The development of commercially available molecular testing for mutations in voltage-gated sodium channels (hyperkalemic periodic paralysis [HyperPP], paramyotonia congenita, and a few hypokalemic periodic paralyses [HypoPP]), L-type calcium channels (majority of HypoPP), and chloride channels (myotonia congenita) has contributed greatly to the clinical evaluation of patients with a suspected channelopathy of skeletal muscle. However, molecular testing is not universally available, it is costly and time-intensive, and it has a false negative rate of at least $20 \%$. The diagnosis of PP or nondystrophic myotonia is therefore still made largely on clinical grounds, supported by electrodiagnostic techniques that were initially described 20 to 50 years ago. On occasion, controlled provocative challenges with ei- 
ther glucose or potassium are performed to differentiate among HypoPP, HyperPP, and normokalemic PP, but these methods are now rarely used. The recognition of certain electrodiagnostic patterns of abnormality may be channel-specific, and perhaps even mutation-specific, thus further guiding molecular analysis. ${ }^{1}$ In the future, these refined electrodiagnostic methods may be used to search for new ion channel mutations, and to help clarify the pathophysiology of these disorders.

The purpose of this review is to summarize some of the currently available methods for the clinical assessment of muscle membrane excitability in channelopathies of skeletal muscle, and to outline their potential applications in diagnosis and treatment of these disorders, as well as determining their natural history. A detailed discussion of the technical aspects and performance of these electrodiagnostic methods is beyond the scope of this review, and the reader is referred to the original sources where appropriate. Finally, although certain diagnostic techniques (e.g., muscle biopsy for the presence of tubular aggregation) are not discussed in this review, because they are less commonly utilized today, the authors acknowledge the role of such techniques in the less common instance where the diagnosis of a muscle channelopathy remains obscure and a more invasive diagnostic procedure is justified.

\section{ELECTRODIAGNOSTIC ASSESSMENT OF MUSCLE MEMBRANE EXCITABILITY}

\section{Exercise testing}

In muscle channelopathies, reduced membrane excitability is manifest clinically by weakness, hypotonia, and hypoflexia and electrodiagnostically by reduced compound muscle action potential (CMAP) amplitude and area. The remarkably dynamic alteration in membrane excitability in response to even brief exercise may result in CMAP changes even in the absence of clinically detectable weakness, thus forming the basis for the short exercise CMAP test initially reported by Streib et al., ${ }^{2}$ and the long exercise CMAP test later reported by McManis et al. ${ }^{3}$ The relative ease of short and long exercise testing using commercially available standard electrodiagnostic equipment has made these the most widely used tests for the assessment of patients with suspected muscle channelopathy.

In both the short ${ }^{2}$ and long exercise tests, ${ }^{3}$ CMAPs are typically recorded from abductor digiti minimi. In the short exercise test, $10 \mathrm{~s}$ of exercise are followed by rest for $60 \mathrm{~s}$. CMAPs are recorded at baseline before exercise, directly after exercise, and then every $10 \mathrm{~s}$ (FIGS. 1A and 1B) for 1 to $2 \mathrm{~min}$. The short exercise test may be repeated successively to increase test sensitivity or specificity still further (see below). In the long exercise test, CMAPs are recorded at baseline before exercise, after each minute during the 5-min exercise period, and then every 1 to $2 \mathrm{~min}$ in the postexercise period for up to 45 min (FIGS. 1C and 1D).

\section{Long exercise test}

The long exercise test is useful for the diagnosis of PP, as well as the nondystrophic myotonia disorders myotonia congenita and paramyotonia congenita. ${ }^{1,3-6}$ The decrement is calculated as the percentage change in CMAP amplitude or area comparing postexercise values to those before or during exercise. Normal cutoffs were derived from healthy controls. McManis et al., ${ }^{3}$ using a cutoff of 40\% decrement in CMAP amplitude and 50\% in CMAP area, reported $71 \%$ sensitivity for the diagnosis of primary PP in a clinically defined cohort of 21 patients with $\mathrm{PP}$, and $100 \%$ specificity against a control group consisting of 14 individuals without PP. There were six positive exercise tests (sensitivity, 67\%) in a cohort of nine patients with secondary causes of PP (e.g., thyrotoxicosis), implying that the specificity of the long exercise CMAP test in differentiating between primary and at least the thyrotoxic form of secondary PP may be relatively poor, a finding that has been confirmed by others. $^{4,6}$

Although most studies have used the McManis exercise protocol, normal cutoffs for postexercise CMAP amplitude decrement have typically been rederived based on local healthy control cohorts, with wide variability: $11 \%$ to $14 \%,{ }^{4} 29 \%,{ }^{6}$ and $41 \%{ }^{5}$ Furthermore, the formula used to calculate CMAP area and amplitude decrements has not been universal. Fournier et al. ${ }^{1}$ compared postexercise CMAP parameters to pre-exercise values (instead of the highest value obtained during or after exercise, as per McManis et al. ${ }^{3}$ ), and used a cutoff of $\geq 20 \%$ drop in CMAP amplitude based on a healthy control group. In their cohort of 27 patients with PP (14 with mutations in the sodium channel and 13 in the calcium channel), they reported that all but one demonstrated an abnormal postexercise CMAP amplitude decrement (i.e., $96 \%$ sensitivity) with $100 \%$ specificity versus healthy controls (FIG. 1D).

Finally, the sensitivity of the long exercise CMAP test appears higher when performed closer to attacks, or before treatment. This is particularly relevant in thyrotoxic PP, in which the likelihood of a positive test declines dramatically once the euthyroid state is achieved. ${ }^{6}$

Given the wide variability in cutoffs that have been used for the long exercise test, an appropriate next step might be to perform receiver-operator characteristic (ROC) analysis in an attempt to offer some standardization of these values to apply universally. In that case, the long exercise CMAP test could be evaluated as a surrogate marker for response in future multicenter clinical trials in channelopathies. 
Arimura et $\mathrm{al}^{4}{ }^{4}$ have attempted to determine the predictive value of the long exercise test in PP associated with low potassium levels. They studied 18 patients-4 of them with primary HypoPP, 7 with thyrotoxic PP, and
7 with other secondary causes of PP (e.g., hyperaldosteronism) - and concluded that the exercise test had excellent sensitivity and negative predictive value for primary $\mathrm{PP}$, given that there were no positive tests in any of their
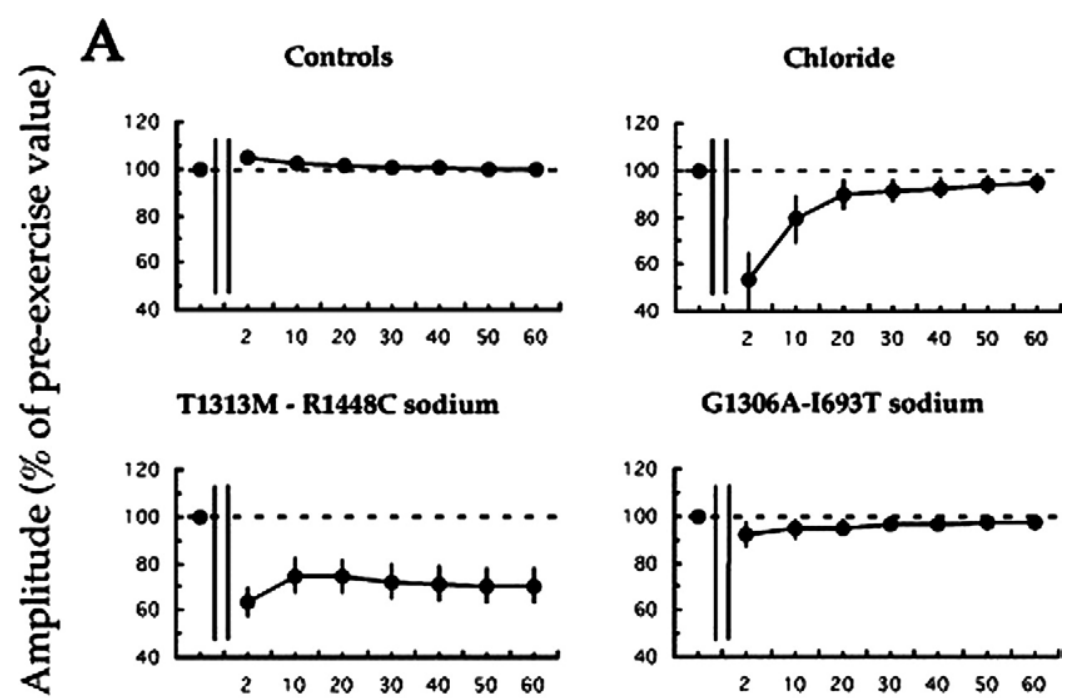

Time after exercise (seconds)
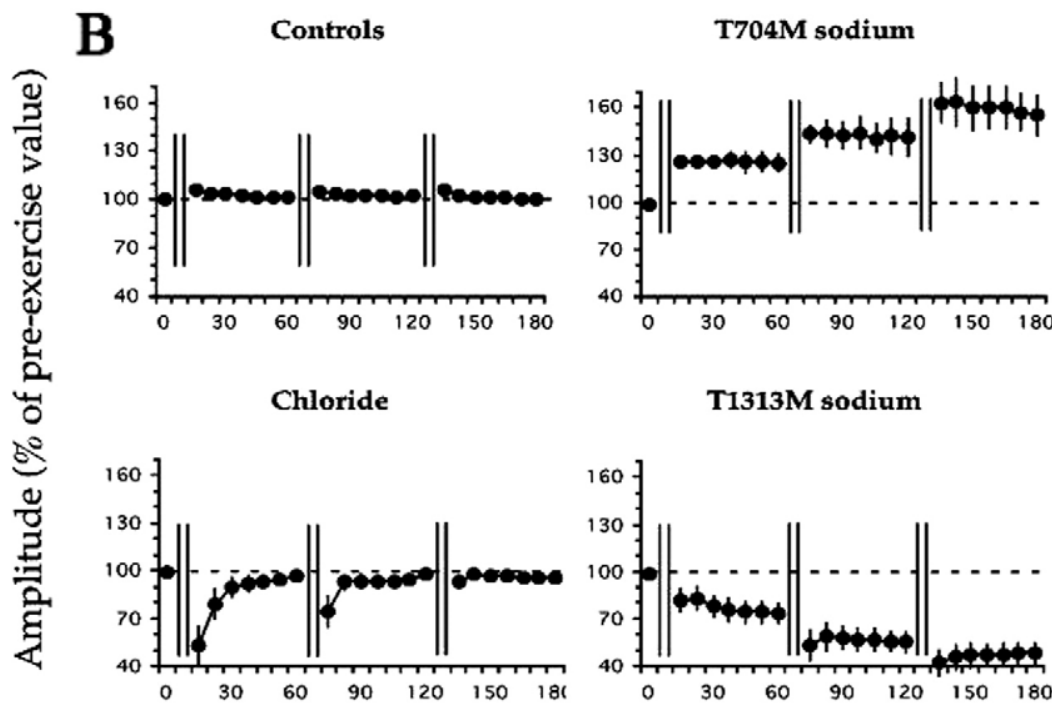

T1313M sodium

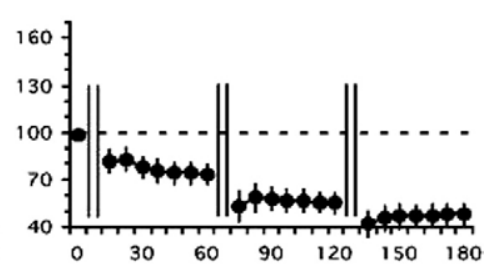

Time after exercise (seconds)

FIG. 1. Electrodiagnostic evaluation of generalized myotonia and periodic paralysis. The amplitude of the compound muscle action potentials (CMAPs), expressed as a percentage of the pre-exercise value, is plotted against the elapsed time after the exercise trial (symbols and vertical bars), mean \pm S.E.M. (A) Short exercise test in myotonic syndromes. Short exercise of the abductor digiti minimi (ADM) muscle, CMAPs recorded during the 50-s resting periods. Changes in CMAP amplitude of ADM muscle following short exercise (double bars) in 41 unaffected controls (top left), 6 myotonia congenita (MC) patients with chloride channel mutations (top right), 6 paramyotonia congenita (PC) patients with T1313M or R1448C sodium channel mutations (bottom left), and 8 patients with G1306A or 1693T sodium channel mutations (bottom right). (B) Effects of short exercise repetition in myotonic syndromes and periodic paralysis in 41 healthy controls (top left), 6 hyperkalemic periodic paralysis (HyperPP) patients with T704M sodium channel mutations (top right), 6 MC patients with chloride channel mutations (bottom left), and 11 PC patients with T1313M sodium channel mutation (bottom right). (C) Long exercise test in myotonic syndromes. Changes in CMAP amplitude of the ADM muscle after long exercise (double bars) in 41 unaffected controls (top left), 6 myotonia congenita (MC) patients with chloride channel mutations (top right), 16 PC patients with T1313M or R1448C sodium channel mutations (bottom left), and 2 patients with G1306A sodium channel mutations (bottom right). (D) Long exercise test in periodic paralysis. Changes in CMAP amplitude of the ADM muscle after long exercise in 6 HyperPP patients with T704M sodium channel mutations (top left), 6 myotonia-HyperPP patients with the I693T mutation of the sodium channel (top right), 13 hypokalemic periodic paralysis 1 (HypoPP-1) patients with the R528H calcium channel mutation (bottom left), and $2 \mathrm{HypoPP}-2$ patients with $\mathrm{R} 672 \mathrm{G}$ or $\mathrm{R} 672 \mathrm{H}$ sodium channel mutations (bottom right). Adapted from Fournier et al. ${ }^{1}$ 

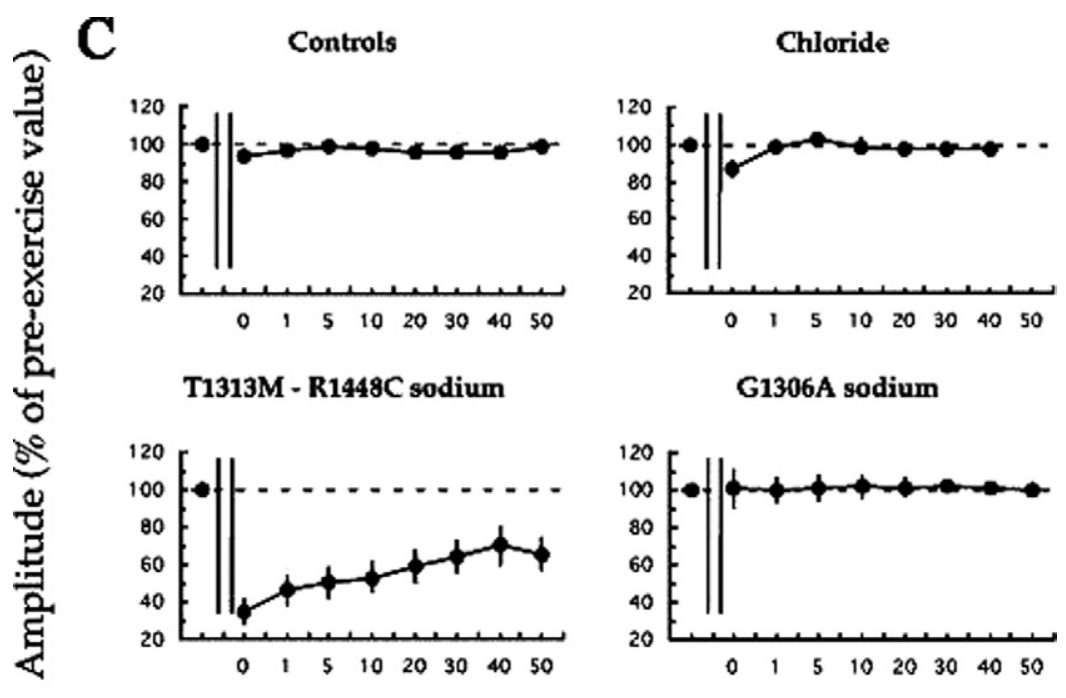

Time after exercise (minutes)
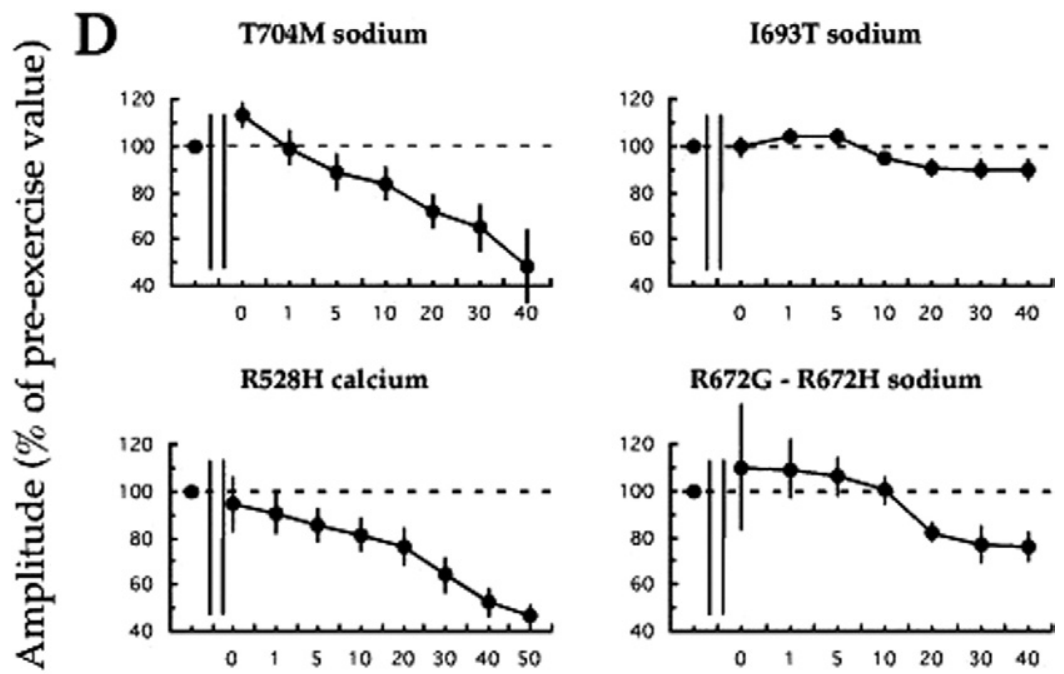

\section{Time after exercise (minutes)}

Fig. 1. (Continued)

secondary PP cases. Several comments are appropriate: 1) the normal cutoff for postexercise CMAP amplitude decrement used in this study was unusually low (14\%), likely enhancing the apparent sensitivity of the test in the study group, 2) a relatively small healthy control group was used (11 subjects) to define their normal cutoffs, possibly making assessment of specificity less reliable, and 3) most of the PP study group were patients with thyrotoxic PP rather than primary PP, limiting the applicability of this study to primary PP.

Regarding other muscle channelopathies, Kuntzer et al. ${ }^{5}$ studied 64 patients: 24 with primary PP (19 with calcium channelopathy and 5 sodium channelopathy, 3 of the latter being paramyotonia congenita and 2 HyperPP), 18 with secondary PP, 6 with myotonia congenita, and 16 with myopathy including myotonic dys- trophy and PROMM. The long exercise test, performed according to McManis et al., ${ }^{3}$ demonstrated an overall $81 \%$ sensitivity for the diagnosis of primary PP (either type ), with $98 \%$ specificity against the normal control group. The sensitivity of the exercise test for the diagnosis of myotonia congenita (17\%) and paramyotonia congenita (33\%) was lower, although the sample size for both groups was small. Therefore, not surprisingly, the exercise test has a higher diagnostic sensitivity for the paralytic phenotype than for the myotonic phenotype, but it is still positive in a minority of the latter. Fournier et al. ${ }^{1}$ confirmed these findings in their cohort of genetically defined patients with chloride (myotonia congenita) and sodium (paramyotonia congenita) channel mutations. Their sensitivity was higher for paramyotonia congenita (100\%), however, and slightly higher also for 
the myotonia congenita group (33\%) — but they used a different normal cutoff as well as a different calculation method for the decrement, as already discussed (FIG. 1C).

In summary, the long exercise test is the most commonly used test in the assessment of suspected muscle channelopathy, with a reported sensitivity ranging from $55 \%$ to $96 \%$ for PP, and $17 \%$ to $100 \%$ for myotonic disorders, the wide variability in part due to equally wide variability in the selection of normal cutoffs among different centers and the method used to calculate these values.

Finally, based on work by Fournier et al., ${ }^{1}$ the sensitivity of the long exercise test may be considerably enhanced by combining exercise CMAP testing with other methodologies, such as concentric needle electromyography or repetitive nerve stimulation at ambient temperature $^{1}$ and after cooling. ${ }^{7}$ In this case, sensitivities may approach $90 \%$ to $100 \%$ in certain populations, while maintaining high specificity, through the recognition of certain patterns that correlate closely with certain genotypes. This approach is promising, and opens an opportunity to confirm the finding in other clinical settings and further expand the correlation between genotype and phenotype in the muscle channelopathies.

\section{Short exercise test}

Streib et al. ${ }^{2}$ originally reported the short exercise test as an alternative to repetitive stimulation in the diagnosis of myotonic disorders, based on the transient paresis and CMAP decrement initially noted 30 years earlier. ${ }^{8}$ Often regarded as simply a shortened and more convenient version of the long exercise test, the short exercise test has been most widely used in the diagnosis of generalized myotonia. Its utility in the diagnosis of PP was highlighted recently by Fournier et al. ${ }^{1}$ (FIGS. 1A and 1B).

In their original study, Streib et al. ${ }^{2}$ evaluated 18 patients with myotonic dystrophy, 3 with recessive myotonia congenita (rMC), 1 with paramyotonia congenita, 10 disease control subjects, 10 healthy control subjects, and 5 asymptomatic relatives. Following exercise, the CMAP amplitude declined in myotonic dystrophy and rMC patients, but the decrement was greater in the latter group. The single patient with paramyotonia congenita demonstrated a prolonged decrement in CMAP amplitude that was still present at $90 \mathrm{~min}$. Overall, the findings were similar in dystrophic and nondystrophic forms of myotonia, but the decrement was more pronounced and more persistent in the latter. This study provided the first evidence in support of an alternative technique to highfrequency repetitive stimulation (see below), which is painful for many patients, and also confirmed that CMAP decrements in these disorders are remarkably invariant across individual patients and across different time frames.
Fournier et al. ${ }^{1}$ extended the study of Streib et al. ${ }^{2}$ by performing three sequential short exercise tests separated by $60 \mathrm{~s}$ (in contrast to the 10-min rest period between repeat tests used in the Streib study). They found a dramatic decline of CMAP amplitude in all 11 patients with $\mathrm{T} 1313 \mathrm{M}$ sodium channel mutations (paramyotonia congenita phenotype) and, by contrast, a progressive increment in six patients with T704M sodium channel mutations (HyperPP phenotype) (FIG. 1A). No CMAP change was noted in those with HypoPP due to calcium channel mutations, but the authors did not comment on whether this was present in the two patients with HypoPP with sodium channel mutations. This provides the most striking example of disparate electrophysiological manifestations of mutations in the same channel (e.g., T1313M vs T704M), albeit with different phenotypes. In six patients with myotonia congenita (four recessive, one dominant, one unknown inheritance), they also noted similar findings to those reported by Streib et al. ${ }^{2}$ : the initial decline in CMAP amplitude following exercise gradually improves with repeated exercise, in a manner similar to the warm-up effect.

\section{SURFACE ELECTROMYOGRAPHY ASSESSMENT OF MUSCLE FIBER CONDUCTION VELOCITY AND POWER SPECTRAL ANALYSIS}

Conduction of action potentials along the muscle fiber membrane is dependent on normal membrane excitability. Thus, quantification of membrane conduction by measurement of muscle fiber conduction velocity (MFCV) holds promise as an ancillary diagnostic test in $\mathrm{PP}$, and as a method to advance our understanding of membrane pathophysiology. Reduction in MFCV in PP is incompletely understood, but may be due to reduction in the membrane length constant as a result of persistent sodium currents and the consequent reduction in transmembrane resistance. ${ }^{9}$

Approximately 25 years ago, Troni et al. ${ }^{10}$ first identified slowing of MFCV both during attacks and in the interictal period in patients with HypoPP. The usefulness of this feature as a diagnostic test in HypoPP has been confirmed by others ${ }^{11-14}$, and its usefulness in electrodiagnosis of generalized myotonia has been reported by Zwarts et al. ${ }^{15}$ Moreover, conduction block of muscle fiber action potentials has been reported in both HypoPP ${ }^{11}$ and in generalized myotonia, ${ }^{16}$ using multichannel surface EMG (see Zwarts and Stegeman for an excellent review). ${ }^{17}$

With respect to HypoPP, reduced MFCV appears to differentiate among healthy controls, affected patients, and even asymptomatic relatives in some cases. Although only a few specific calcium channel mutations in HypoPP have been studied, the basic pathophysiology 

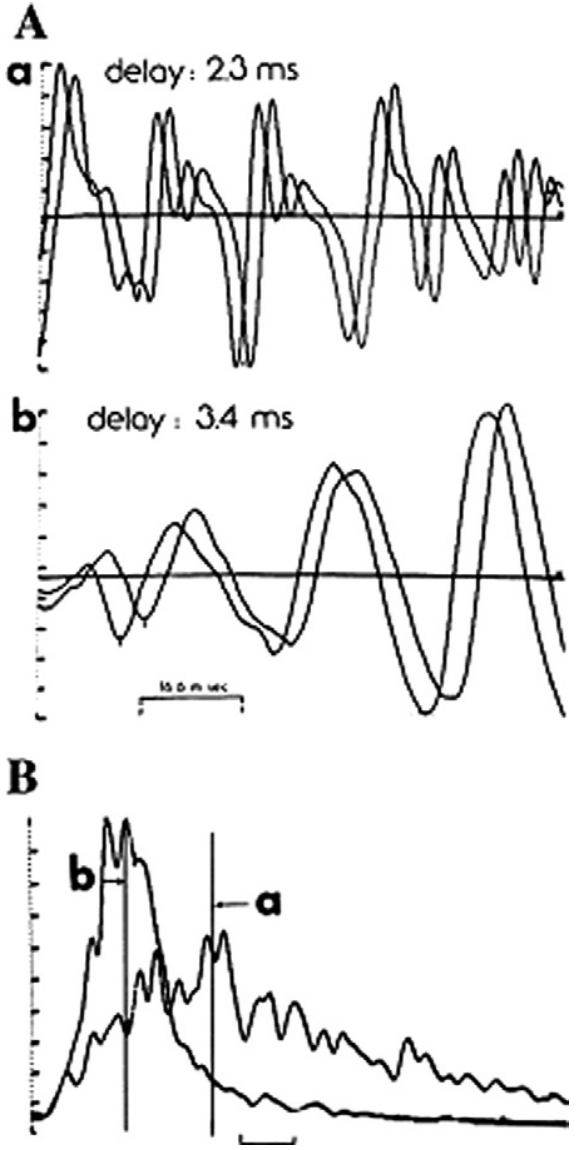

FIG. 2. Muscle fiber conduction velocity (MFCV) and power spectra in HypoPP. (A) Examples of the normal (a) and reduced (b) MFCV in a family with HypoPP. The calculated MFCV in 4.4 and $2.8 \mathrm{~m} \cdot \mathrm{s}^{-1}$, the cross-correlation coefficients are 0.95 (a) and 0.97 (b), respectively. The vertical calibration is $0.24 \mathrm{mV}$ per division. Note the much lower frequency content of the signals from the patient. (B) The power spectra of the measurements in (A). The median frequency $F_{\text {med }}$ of signal (a) is $86 \mathrm{~Hz}$, the $F_{\text {med }}$ of signal (b) is $44 \mathrm{~Hz}$. The horizontal calibration is $24.4 \mathrm{~Hz}$. Reprinted from Zwarts et al., ${ }^{14}$ with permission.

underlying PP suggests that reduced MFCV may be a universal finding in HypoPP, if not necessarily in all forms of PP. Unfortunately, the applicability of this technique is limited by the requirement for highly trained personnel and complex multichannel EMG equipment, which is typically available in only a few specialist centers.

A more simplified approach was proposed by Zwarts et al., ${ }^{14}$ who used a linear array of three electrodes to record surface EMG from the biceps in healthy controls, 10 individuals with HypoPP, and 15 of their asymptomatic relatives (FIG. 2A). Mean MFCV in normal subjects was $4.55 \pm 0.33 \mathrm{~m} / \mathrm{s}$, compared with 3.37 $\pm 0.35 \mathrm{~m} / \mathrm{s}$ in those affected $(P<0.001)$; in $8 / 9$ patients, the MFCV was more than 2 S.D. below that of healthy controls (sensitivity, 89\%). In addition, MFCV was reduced in 6/15 asymptomatic relatives, 2 of whom were found to have a vacuolar myopathy on muscle biopsy.
Unfortunately, molecular confirmation of PP in this cohort was unavailable at the time, and it would be of interest to repeat this analysis in a genetically defined cohort to correlate reduced MFCV to genotype.

Van der Hoeven et al. ${ }^{13}$ reported similar findings in a cohort of 33 HypoPP carriers (also clinically but not molecularly defined), and 56 of their asymptomatic relatives, using surface EMG (s-MFCV) recordings from the biceps. Approximately one third of each group then underwent invasive EMG (i-MFCV) recording using an intramuscular stimulating electrode, with a concentric needle recording electrode placed $10 \mathrm{~mm}$ to $15 \mathrm{~mm}$ distally in the biceps (the exact location directed by the evoked twitch). Mean MFCV measured using s-MFCV was $4.25 \pm 0.37 \mathrm{~m} / \mathrm{s}$ in normal subjects, $3.36 \pm 0.22 \mathrm{~m} / \mathrm{s}$ in HypoPP patients, and $4.54 \pm 0.52 \mathrm{~m} / \mathrm{s}$ in their asymptomatic relatives. Using a cutoff defined by 2 S.D. below the mean (i.e., $<3.51 \mathrm{~m} / \mathrm{s}$ ), the sensitivity for the carrier state was $77 \%$ using s-EMG. For i-MFCV, the values in the normal group were somewhat lower than that for s-MFCV at $3.17 \pm 0.22 \mathrm{~m} / \mathrm{s}$, and were $2.36 \pm 0.31 \mathrm{~m} / \mathrm{s}$ in the HypoPP group, and $3.15 \pm 0.51 \mathrm{~m} / \mathrm{s}$ in their asymptomatic relatives. Based on the finding of increased dispersion in MFCV (i.e., an increased range of individual fiber conduction velocities) in HypoPP, the authors plotted i-MFCV against a ratio of the fastest to slowest conduction velocities recorded from several fibers (F/S ratio) and were able to differentiate carriers from healthy controls with $100 \%$ sensitivity, although the specificity of this finding was not calculated. Although this study clearly indicates that MFCV measurement using invasive or surface techniques is moderately sensitive in identifying those with HypoPP and probably also asymptomatic HypoPP carriers, the sensitivity and specificity of MFCV in a genetically defined cohort is unknown. This question could be definitively addressed by comparing a genetically defined cohort with asymptomatic relatives to determine the predictive value of MFCV testing in identifying carrier status, and might be an appropriate direction for future studies. In conclusion, invasive and surface MFCV techniques have relative advantages and disadvantages, and both probably have a role in diagnosis of PP.

Analysis of surface EMG frequency content (power spectra) also holds promise as a useful diagnostic tool, given that frequency content is closely related to MFCV, but also depends on morphology of individual recorded motor unit potentials, their position relative to the recording electrode, and the configuration and dimensions of recording electrode itself (FIG. 2B). EMG power spectral analysis is advantageous in that does not require complex multichannel EMG equipment, because the frequency content can be decomposed from the EMG signal recorded from as few as two electrodes using a standard EMG amplifier. However, signal processing techniques 
to extract the frequency content and calculate power spectra make several assumptions about the EMG signal, depending on the method used.

The most widely used method, the fast Fourier transform (FFT), assumes that the signal is stationary (i.e., composed of multiple individual but nonvariant frequencies over time). Over short time epochs (500 ms to $2 \mathrm{~s}$ ) during isometric contractions, this is probably a reasonable assumption, but does not hold true for longer epochs (e.g., continuous contraction for several seconds). ${ }^{18}$ The second major limitation of FFT is that it provides an average of frequency composition over the entire duration of the signal, rather than resolving frequency content at specific timepoints during the signal. This is particularly an issue in situations where the frequency content of a signal changes over time (e.g., reduced median frequency during fatiguing contractions). Instead, the short-time Fourier transform (STFT) has been used in studies of muscle fatigue during sustained contractions. In this instance, FFT is performed on individual short epochs after decomposition of the EMG signal into its time components, thereby giving a representation of frequency content over a defined period (e.g., 2-s windows during a sustained contraction at $50 \%$ MVC for $30 \mathrm{~s}$ ). The technique could probably be adapted relatively easily for study of the muscle channelopathies, where dynamic variation in muscle excitability thresholds during exercise in individuals with PP might reveal diagnostically useful alterations in power spectral characteristics. $^{17,19}$

\section{REPETITIVE NERVE STIMULATION STUDIES AND TRANSIENT PARESIS}

Decrements in CMAP amplitude and area on repetitive nerve stimulation were identified in both recessive (Becker) and dominant (Thomsen) myotonia congenita more than 30 years ago, typically using higher repetitive stimulation frequencies than those used in the diagnosis of neuromuscular junction disorders. In contrast to the neuromuscular junction disorders, CMAP amplitude decrements in myotonia congenita do not improve with cholinesterase inhibition and persist even when the muscle is stimulated directly (thereby bypassing the neuromuscular junction). The presence and degree of CMAP decrement on repetitive nerve stimulation in myotonia congenita appears to be related to mutation type: it is universal with rMC, but less frequent in $\mathrm{dMC}$ (dominant myotonia congenita). ${ }^{20}$ Furthermore, among dominant mutations there is considerable heterogeneity in CMAP amplitude decrements (FIG. 3).

The CMAP decrement with repetitive stimulation resolves with continued exercise, resembling the warm-up phenomenon typical of myotonia congenita. Interestingly, the presence and severity of this decrement does
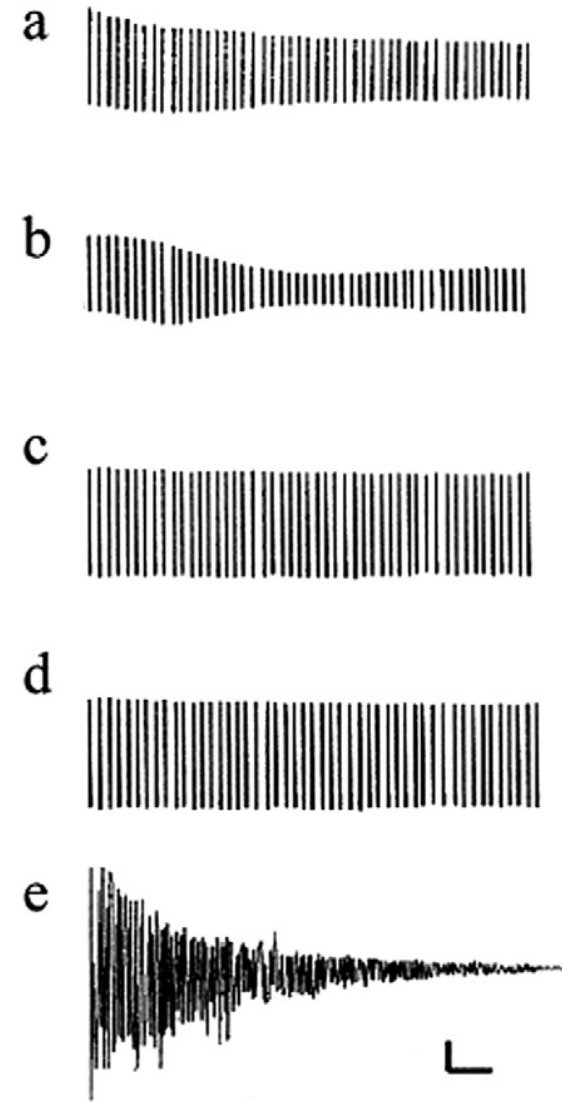

FIG. 3. Electrophysiological findings in five patients with myotonia congenita. (a) Decrement in patient 1 (mutation P480L, Dr. Thomsen's family). (b) Decrement in patient 4 (mutation P480L, Dr. Thomsen's family). In this patient, the decrement was maximal after approximately 25 stimulations, after which the amplitude increased. (c) Absence of decrement in patient 16 (mutation M128V). (d) Absence of decrement in patient 19 (mutation E193K). In (a-d), the vertical scaling was $5 \mathrm{mV}$ per division. The horizontal scaling was $0.5 \mathrm{~s}$ per division ( 5 pulses per division). (e) Electromyographic (EMG) activity after maximal voluntary contraction (patient 5, Dr. Thomsen's family). The duration of EMG activity was $9.9 \mathrm{~s}$. Vertical scaling was $100 \mu \mathrm{V}$ per division and horizontal scaling was $1 \mathrm{~s}$ per division. Reprinted from Colding-Jørgensen et al., ${ }^{20}$ with permission.

not appear to correlate well with the severity of myotonia as reported by patients, ${ }^{21,22}$ but does correlate quite well with the degree of transient paresis measured by force transducers $^{23}$ and, at least for dominant CLCN1 mutations, also with the degree of reduction in chloride conductance (FIG. 4). ${ }^{24}$ Clearly, myotonia and transient paresis are closely allied in these disorders, but the pathophysiologic link between the two phenomena remains poorly understood.

Rudel et al. ${ }^{25}$ demonstrated that in some patients with rMC, the clinical response to tocainide was paralleled by a reduction in transient paresis, suggesting that quantification of this phenomenon may be a useful surrogate marker for therapeutic response in clinical trials. However, the lack of uniformity in the presence of transient paresis among common mutations in myotonia congenita 

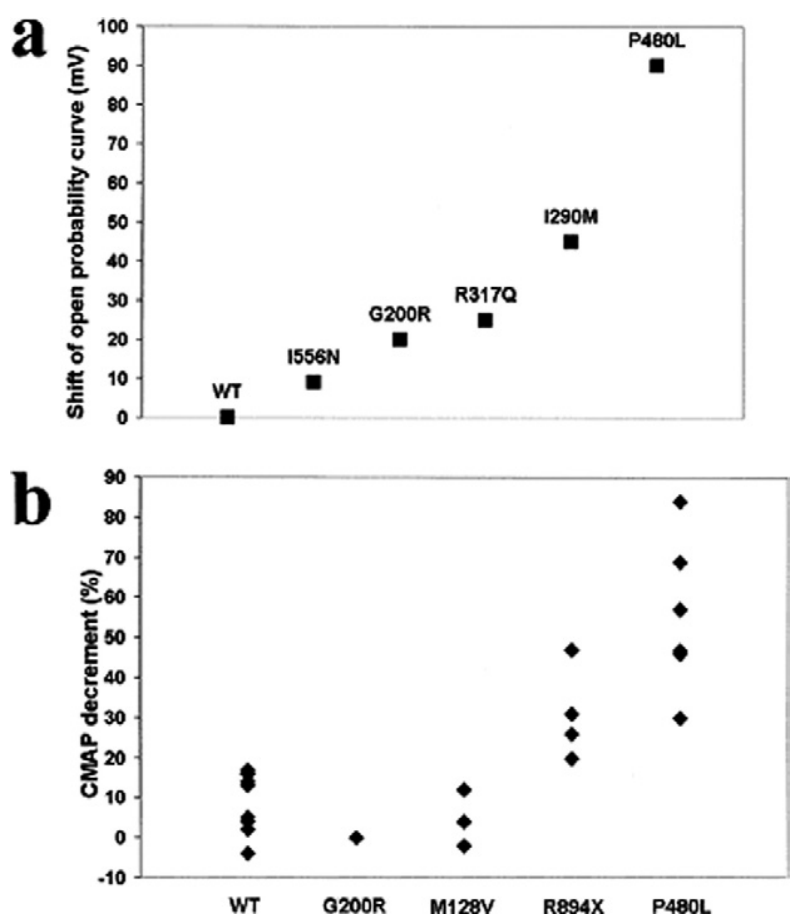

FIG. 4. Some phenotypic variability reflects different mutation types. (a) Dominant CLCN1 mutations cause different degrees of chloride conductance reduction. A 1:1 expression of wild-type (WT) and mutant RNA in oocytes yields different degrees of chloride conductance reduction, expressed here as the shift of voltage dependence of the open probability curve (Kubisch et al., ${ }^{41}$ Pusch et al., ${ }^{42}$ Wollnik et al. ${ }^{43}$ ). It is seen that $1: 1$ expression of the $1556 \mathrm{~N}, \mathrm{G} 200 \mathrm{R}$, and R317Q mutations causes only slight to moderate gating abnormalities, in accordance with the mild phenotype of patients heterozygous for these mutations. In contrast, a 1:1 expression of I290M and P480L causes severe gating abnormalities, corresponding to the full penetrance and often pronounced myotonia of heterozygous patients. (b) Dominant mutations cause different degrees of CMAP decrement at $10-\mathrm{Hz}$ repetitive nerve stimulation (ColdingJørgensen et al., ${ }^{20}$ Deymeer et al. ${ }^{26}$ ). No decrement is observed in patients heterozygous for the 'mild' G200R mutation, whereas a pronounced decrement is seen in patients heterozygous for the P480L mutation. Reprinted from Colding-Jørgensen et al., ${ }^{24}$ with permission.

poses a significant problem for this approach. Furthermore, although CMAP amplitude decrements are remarkably reproducible in the same patient at different times, ${ }^{20-22}$ there is marked variability among different family members carrying the same mutation, presumably due to environmental factors ${ }^{20}$ or, perhaps, to differential allelic expression. ${ }^{24}$

The optimum frequency for repetitive nerve stimulation in these disorders has been studied, but is not well established. Various stimulation paradigms have been proposed. Probably the most widely used is a frequency of $10 \mathrm{~Hz}$ for $5 \mathrm{~s}$. Regarding the sensitivity and specificity using this method, Deymeer et al. ${ }^{26}$ evaluated a cohort of 25 patients with rMC, 6 with dMC, 1 with paramyotonia congenita, and 1 with HyperPP. Their sensitivity was $96 \%$ for rMC using a decrement cutoff of $\geq 25 \%$, with $88 \%$ specificity in differentiating rMC from other forms of nondystrophic myotonia (dMC, HyperPP, or paramyotonia congenita). Normal and other neuromuscular disease control subjects were not studied, however, and the sample size for the nondystrophic myotonia group comprising nonrecessive forms was small, thus partly limiting the strength of the study.

More recently Colding-Jørgensen et al. ${ }^{20}$ used $10-\mathrm{Hz}$ repetitive nerve stimulation for $5 \mathrm{~s}$ in a study of 20 subjects with myotonia congenita ( 15 with $\mathrm{dMC}, 3$ with rMC, and 2 asymptomatic relatives carrying the R894X CLCN1 mutation) and 11 normal subjects. They also used a cutoff of $\geq 25 \%$, but in this case it was defined by a value of 2 S.D. above the mean decrement in their normal control cohort. They found a wide range of CMAP amplitude changes in $\mathrm{dMC}$ ( $1 \%$ increment to $86 \%$ decrement). Certain dominant mutations were particularly associated with a CMAP amplitude decrement of $>25 \%$ : P480L (all 6 cases), and R894X ( 3 of 6 cases in which decrement was calculated) (FIG. 3).

Fournier et al. ${ }^{1}$ reported a different approach with ulnar nerve $3-\mathrm{Hz}$ repetitive stimulation for a total of 10 stimuli following short exercise. They observed a $74 \%$ decrement in CMAP amplitude on repetitive nerve stimulation after short exercise, $10 \mathrm{~s}$ in duration, in a subject with paramyotonia congenita carrying the R1448C sodium channel mutation.

In summary, repetitive nerve stimulation offers high sensitivity and negative predictive value for the recessive forms of myotonia congenita and fairly high sensitivity for dominant forms of myotonia congenita. The utility in differentiating between $\mathrm{dMC}$ and $\mathrm{rMC}$ is poorly characterized; it is probably dependent on mutation type. Finally, there is a lack of standardization of repetitive stimulation protocols across centers. Although $10-\mathrm{Hz}$ stimulation for $5 \mathrm{~s}$ appears to be the best studied, it may also be less well tolerated than lower stimulation frequencies in some patients, and further study of lower stimulation frequencies, with or without prior short exercise, seems appropriate.

\section{QUANTIFICATION OF MYOTONIA}

Although bedside and needle electromyography can reliably determine the presence of myotonia, they do not as easily allow for its quantification. Therefore, several groups have used relaxation time of a voluntary ${ }^{27-30}$ or evoked $^{28,31-33}$ isometric muscle contraction recorded with a force transducer as a measure of myotonia (FIG. 5). The advantages of using relaxation time of a twitch or tetanic stimulation over voluntary activation (e.g., maximal handgrip ${ }^{27-30}$ ) are that this approach requires less patient cooperation, is independent of central nervous system input (e.g., descending voluntary commands to relax voluntarily activated muscles), and is not contaminated by voluntary activation of antagonist mus- 
Twitch Recordings

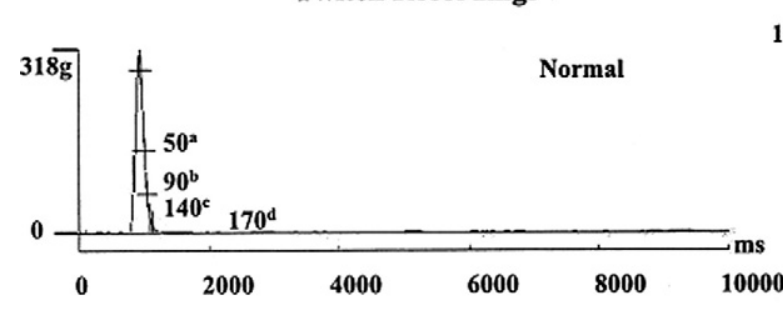

Tetanic Recordings

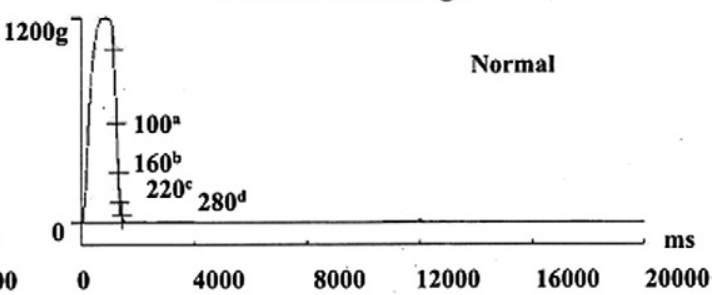

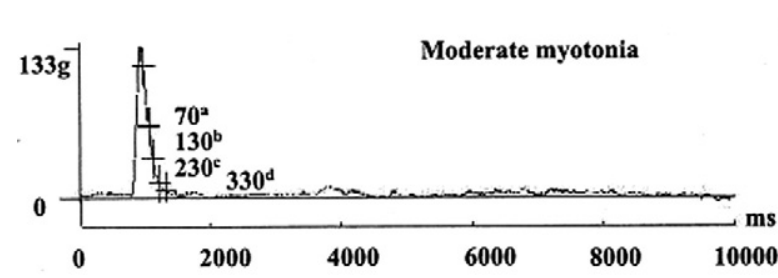
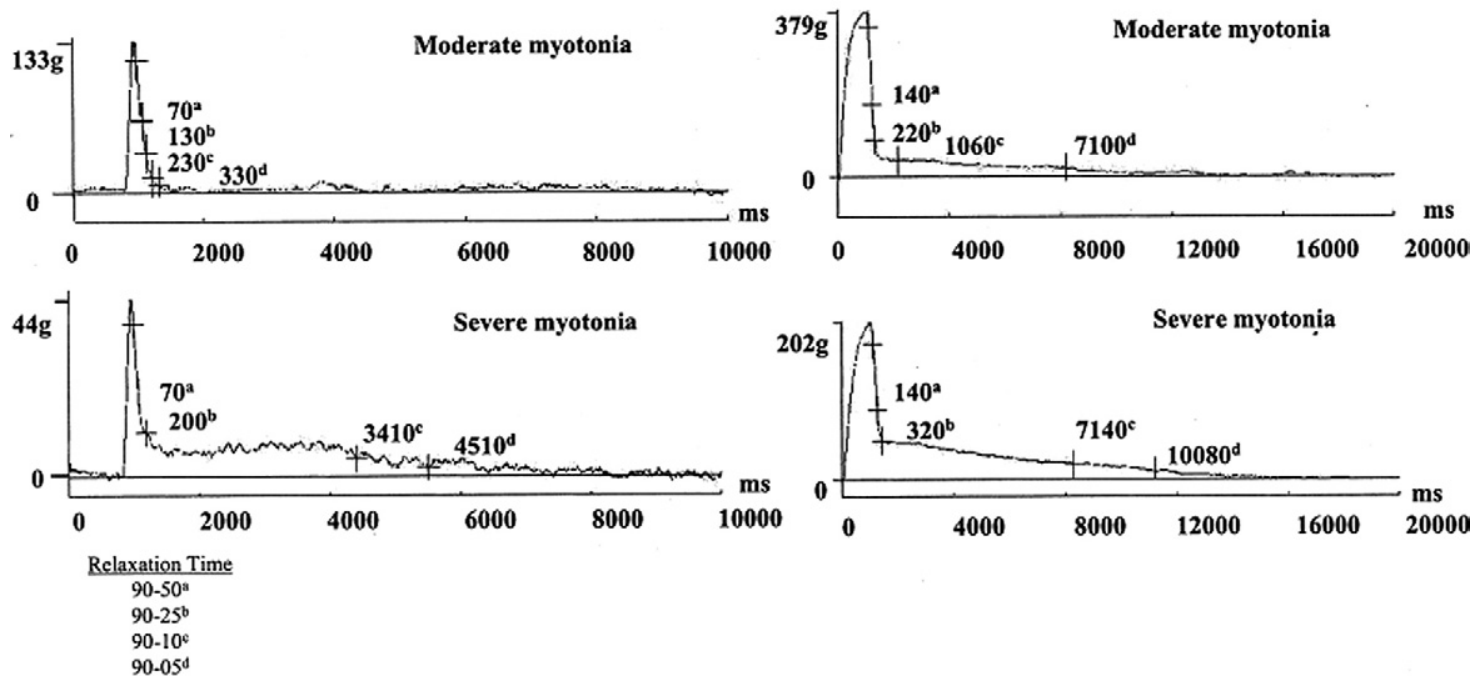

FIG. 5. Twitch (left) and tetanic (right) relaxation times (RT) on the $y$-axis, with log scale from 0 to $10,000 \mathrm{~ms}$ (left) and 0 to $100,000 \mathrm{~ms}$ (right), plotted against peak forces $g$ on the $x$-axis: $90 \%$ to $5 \%$ RT (top), $90 \%$ to $50 \%$ RT (middle), and $50 \%$ to $5 \%$ RT (bottom) in subjects (triangles) and myotonic dystrophy type 1 (DM1) patients (squares). The mean + 2.5 S.D. for the normal subjects (the normal upper limit) is shown as a dotted line. Note that for both the twitch and the tetanic data, 1) the highest peak force seen in the DM1 group is still lower than the lowest peak force seen in the normal group; 2) the $90 \%$ to $5 \%$ RT is much longer in the DM1 patients than in the subjects, and all but four DM1 patients have a twitch $90 \%$ to $5 \%$ RT above the normal limit and all but two DM1 patients have a tetanic $90 \%$ to $5 \%$ RT above the normal limit; 3) for both twitch and tetanic contractions, the terminal phase of muscle relaxation (50\% to $5 \%$ RT) rather than the initial phase (90\% to $50 \%$ RT) accounts for most of the delay in $90 \%$ to $5 \%$ RT; and 4) compared with twitch RT, tetanic RT are disproportionately prolonged in the patients than in the subjects. Reprinted from Logigian et al., ${ }^{28}$ with permission.

cles (e.g., voluntary use of finger extensors to open a myotonically closed hand). The disadvantages of evoked responses for this purpose are that stimulations can be painful and also that a more complicated technical set-up is required.

Quantification of relaxation time using either method can provide a surrogate clinical marker to follow the natural history of myotonia in patients with myotonic disorders, to determine the efficacy of antimyotonia treatment (e.g., mexilitine) and to assess genetic-physiologic correlations ${ }^{28}$ and the mechanisms of transient paresis and warm-up of myotonia. ${ }^{27}$

\section{PROVOCATIVE CHALLENGES IN THE DIAGNOSIS OF MUSCLE CHANNELOPATHIES}

Intake of glucose in HypoPP and potassium in HyperPP, potassium-aggravated myotonia, or paramyotonia congenita often provoke attacks of paralysis or stiffness in patients with PP and nondystrophic myotonia. Second, both oral and intravenous potassium loading shorten paralytic attack duration in HypoPP. ${ }^{34,35}$ Most studies of glucose/potassium loading in PP were conducted before the advent of molecular testing and when electrodiagnostic testing for PP was relatively limited; provocative challenges now are rarely performed. Nonetheless, systematic measurement of metabolic parameters before and during attacks has contributed substantially to our understanding of the pathophysiology of these disorders. No studies have directly addressed the question of diagnostic sensitivity/specificity of provocative testing in a cohort of patients with suspected muscle channelopathy. Most studies report qualitative effects of loading on strength, and document classification of PP as either HypoPP or HyperPP through precipitation of attacks with glucose or glucose/insulin, epinephrine, or potassium. ${ }^{36,37}$ In two individuals with normokalemic PP neither potassium nor glucose precipitated attacks. ${ }^{38}$ Both had vacuoles and collections of tubular aggregates on muscle biopsy, and the clinical features were similar to those of a cohort of patients who were later identified as having a sodium channel mutation associated with 
HyperPP. ${ }^{39}$ Thus, those with normokalemic PP are probably a subset of PP associated with sodium channel mutations, and glucose or potassium loading may be of lower sensitivity in this setting. Such patients appear less likely to respond to acetazolamide, and may even worsen on it, for unclear reasons.

With respect to the administration of glucose as a hypokalemic stimulus, the concomitant administration of insulin is probably unnecessary, because in most cases glucose stimulates sufficient release of endogenous insulin to provoke hypokalemia. ${ }^{40}$

For the hyperkalemic challenge or in the treatment of HypoPP, oral rather than intravenous potassium supplementation is probably safer, given the risk of rebound hyperkalemia in patients with HypoPP who have impaired potassium homeostasis, compared with healthy individuals and those with secondary HypoPP (e.g., hyperaldosteronism or thyrotoxicosis). If intravenous loading is required, mannitol-based rather than glucose-based diluents may be preferable, because the glucose in the latter appears to partially offset the potassium-repleting effect, presumably by stimulation of endogenous insulin release. $^{34}$

In summary, both oral and intravenous loading with glucose or potassium are effective in precipitating attacks in HypoPP and HyperPP, respectively, but oral loading is probably safer and less likely to be associated with rebound hyperkalemia or hyperglycemia. The use of such loading has diminished in the present molecular era, but may continue to have a role when molecular testing is unavailable or if biochemical clarification of the type of PP is required. The sensitivity of provocative challenges in the setting of normokalemic PP is probably less than for other types of PP. Finally, whether the diagnostic sensitivity and specificity of electrodiagnostic testing can be enhanced if performed in the setting of a provocative challenge remains unanswered but might be an appropriate avenue for future clinical trials.

\section{CLINICAL TRIALS IN MUSCLE CHANNELOPATHIES}

Although our understanding of the skeletal muscle channelopathies has advanced dramatically over the past 30 years, based on development of molecular diagnosis and refinement of electrodiagnostic testing, considerable work remains to be done in correlating phenotype and genotype in this group of disorders, and several questions remain. For example, why do members of the same family who carry the same mutation manifest vastly different phenotypes?

Although we have existing and effective treatments in many cases, it is clear that their efficacy is not universal and some individuals may paradoxically worsen on them. Furthermore, initially effective treatments may lose their efficacy over time, for unclear reasons. The need for accurate clinical characterization and effective ancillary testing has never been greater for diagnosis of genetically negative or undefined cases and for accurate clinical description of new mutations. The presence of incomplete penetrance in certain channelopathies (particularly in women with calcium channel mutations associated with HypoPP) poses another diagnostic challenge, and new nonmolecular diagnostic techniques such as electrodiagnosis may be particularly informative in this situation. For all these reasons, continued clinical, electrodiagnostic, and molecular research is vital to our progress in recognizing, understanding, and treating patients with these interesting disorders.

Acknowledgments: The authors thank Jamie Bentivegna for assistance in preparation of the manuscript.

\section{REFERENCES}

1. Fournier E, Arzel M, Sternberg D, et al. Electromyography guides towards subgroups of mutations in muscle channelopathies. Ann Neurol 2004;56:650-661.

2. Streib EW, Sun SF, Yarkowsky T. Transient paresis in myotonic syndromes: a simplified electrophysiologic approach. Muscle Nerve 1982;5:719-723.

3. McManis PG, Lambert EH, Daube JR. The exercise test in periodic paralysis. Muscle Nerve 1986;7:704-710.

4. Arimura Y, Arimura K, Suwazono S, et al. Predictive value of the prolonged exercise test in hypokalemic paralytic attack. Muscle Nerve 1995;18:472-474.

5. Kuntzer T, Flocard F, Vial C, et al. Exercise test in muscle channelopathies and other muscle disorders. Muscle Nerve 2000;23: 1089-1094.

6. Tengan CM, Antunes AC, Gabbai AA, Manzano GM. The exercise test as a monitor of disease status in hypokalemic periodic paralysis. J Neurol Neurosurg Psychiatry 2004;75:497-499.

7. Fournier E, Viala K, Gervais H, et al. Cold extends electromyography distinction between ion channel mutations causing myotonia. Ann Neurol 2006;60:356-365.

8. Lambert EH, Millikan CH, Eaton LM. Stage of neuromuscular paralysis in myotonia. Am J Physiol 1952;171:741.

9. Rudel R, Lehmann-Horn F, Ricker K, Kuther G. Hypokalemic periodic paralysis: in vitro investigation of muscle fiber membrane parameters. Muscle Nerve 1984;7:110-120.

10. Troni W, Doriguzzi C, Mongini T. Interictal conduction slowing in muscle fibers in hypokalemic periodic paralysis. Neurology 1983; 33:1522-1525.

11. Links TP, van der Hoeven JH, Zwarts MJ. Surface EMG and muscle fiber conduction during attacks of hypokalemic periodic paralysis. J Neurol Neurosurg Psychiatry 1994;57:632-634.

12. Links TP, van der Hoeven JH. Muscle fiber conduction velocity in $\arg 1239$ his mutation in hypokalemic periodic paralysis. Muscle Nerve 2000;23:296.

13. Van der Hoeven JH, Links TP, Zwarts MJ, van Weerden TW. Muscle fiber conduction velocity in the diagnosis of familial hypokalemic periodic paralysis: invasive versus surface determination. Muscle Nerve 1994;17:898-905.

14. Zwarts MJ, van Weerden TW, Links TP, Haenen HTM, Oosterhuis HJG. The muscle fiber conduction velocity and power spectra in familial hypokalemic periodic paralysis. Muscle Nerve 1988;11: $166-173$

15. Zwarts MJ, van Weerden TW. Transient paresis in myotonic syndromes. Brain 1989;112:665-680.

16. Drost G, Blok JH, Stegeman DF, van Dijk JP, van Engelen BGM, Zwarts MJ. Propagation disturbance of motor unit action potentials 
during transient paresis in generalized myotonia: a high density surface EMG study. Brain 2001;124:352-360.

17. Zwarts MJ, Stegeman DF. Multichannel surface EMG: basic aspects and clinical utility. Muscle Nerve 2003;28:1-17.

18. Zazula D, Karlsson S, Doncarli C. Advanced signal processing techniques. In: Merletti R, Parker PA, editors. Electromyography: physiology, engineering, and noninvasive applications. Piscataway, NJ: IEEE Press; 2004:259-304.

19. Wakeling JM, Pascual SA, Nigg BM, von Tscharner V. Surface EMG shows distinct populations of muscle activity when measured during sustained submaximal exercise. Eur J Appl Physiol 2001;86:40-47.

20. Colding-Jørgensen E, Duno M, Schwartz M, Vissing J. Decrement of compound muscle action potential is related to mutation type in myotonia congenita. Muscle Nerve 2003;27:449-455.

21. Aminoff MJ, Layzer RB, Satya-Murti S, Faden AI. The declining electrical response of muscle to repetitive nerve stimulation in myotonia. Neurology 1977;27:812-816.

22. Brown JC. Muscle weakness after rest in myotonic disorders: an electrophysiologic study. J Neurol Neurosurg Psychiatry 1974;37: $1336-1342$

23. Ricker K, Haass A, Hertel G, Mertens HG. Transient muscle weakness in severe recessive myotonia congenita: improvement of isometric force by drugs relieving myotonic stiffness. J Neurol 1978;218:253-262.

24. Colding-Jørgensen E. Phenotypic variability in myotonia congenita. Muscle Nerve 2005;32:19-34.

25. Rudel R, Ricker K, Lehmann-Horn F. Transient weakness and altered membrane characteristic in recessive generalized myotonia (Becker). Muscle Nerve 1988;11:202-211.

26. Deymeer F, Cakirkaya S, Serdaroglu P, et al. Transient weakness and compound muscle action potential decrement in myotonia congenita. Muscle Nerve 1998;21:1334-1337.

27. Logigian EL, Blood CL, Dilek N, et al. Quantitative analysis of the "warm-up" phenomenon in myotonic dystrophy type 1. Muscle Nerve 2005;32:35-42.

28. Logigian EL, Moxley RT 4th, Blood CL, et al. Leukocyte CTG repeat length correlates with severity of myotonia in myotonic dystrophy type 1 . Neurology 2004;62:1081-1089.

29. Ricker K, Lehmann-Horn F, Moxley RT 3rd. Myotonia fluctuans. Arch Neurol 1990;47:268-272.

30. Torres C, Moxley RT, Griggs RC. Quantitative testing of handgrip strength, myotonia, and fatigue in myotonic dystrophy. J Neurol Sci 1983;60:157-168.
31. McComas AJ, Campbell MJ, Sica REP. Electrophysiological study of dystrophia myotonica. J Neurol Neurosurg Psychiatry 1971;34: 132-139.

32. Taylor RG, Abresch RT, Lieberman JS, Fowler WM Jr, Entrikin RK. Analysis of human muscle contractility with a microcomputercontrolled stimulus and data acquisition system. Arch Phys Med Rehabil 1992;73:228-232.

33. Taylor RG, Abresch RT, Lieberman JS, Fowler WM Jr, Entrikin RK. In vivo quantification of muscle contractility in humans: healthy subjects and patients with myotonic muscular dystrophy. Arch Phys Med Rehabil 1992;73:233-236.

34. Griggs RC, Resnick J, Engel WK. Intravenous treatment of hypokalemic periodic paralysis. Arch Neurol 1983;40:539-540.

35. Manary M, Keating JP, Hirshberg GE. Quadriparesis due to potassium depletion. Crit Care Med 1986;14:750-752.

36. Jarrell MA, Greer M, Maren TH. The effect of acidosis in hypokalemic periodic paralysis. Arch Neurol 1976;33:791-793.

37. Minaker KL, Meneilly GS, Flier JS, Rowe JW. Insulin-mediated hypokalemia and paralysis in familial hypokalemic periodic paralysis. Am J Med 1988;84:1001-1006.

38. Meyers KR, Gilden DH, Rinaldi CF, Hansen JL. Periodic muscle weakness, normokalemia, and tubular aggregates. Neurology 1972; 22:269-279.

39. Chinnery PF, Walls TJ, Hanna MG, Bates D, Fawcett PRW. Normokalemic periodic paralysis: does it exist? Ann Neurol 2002;52: 251-252.

40. Johnsen T. A new standardized and effective method of inducing paralysis without administration of exogenous hormone in patients with familial periodic paralysis. Acta Neurol Scand 1976;54:167172.

41. Kubisch C, Schmidt-Rose T, Fontaine B, Bretag AH, Jentsch TJ $\mathrm{ClC}-1$ chloride channel mutations in myotonia congenita: variable penetrance of mutations shifting the voltage dependence. Hum Mol Genet 1998;7:1753-1760.

42. Pusch M, Steinmeyer K, Koch MC, Jentsch TJ. Mutations in dominant human myotonia congenita drastically alter the voltage dependence of the ClC-1 chloride channel. Neuron 1995;15:14551463.

43. Kubisch C, Steinmeyer K, Pusch M. Identification of functionally important regions of the muscular chloride channel $\mathrm{C} 1 \mathrm{C}-1$ by analysis of recessive and dominant myotonic mutations. Hum Mol Genet 1997;6:805-811. 